Rev. Elev Méd. vét. Pays trop., 1970, 23 (2) : 243-48

\title{
Note sur la composition en acides-aminés de crevettes pouvant être utilisées dans l'alimentation des animaux d'élevage à Madagascar
}

\author{
par R. GAULIER, \\ Pharmacien-Chimiste en Chef de $2^{\mathrm{e}}$ Classe \\ avec la collaboration technique de Madame F. ALEXANDRE
}

\begin{abstract}
RESUME
Certains crustacés que lon trouve en abondance à Madagascar pourraient être utulisés dans l'alimentation arimale, à la place de farines de poisson d'importation :

- Petites crevettes d'eau douce: Caridina nilotica xiphias Atyidae;

- Petites crevettes de mer: Sergestes arcticus, Penaesdae.

Il en est de même pour les céphalothorax de grosses crevettes de mer (Penaeus indicus...) qui constıtuent des déchets de la pêche industrielle dans la région de Majunga.

La composition chimique globale et la composition ел acides-aminés de ces 3 produits sont indiquées dans cet article.
\end{abstract}

\section{INTRODUCTION}

Le problème de l'alimentation azotée des animaux d'élevage à Madagascar est bien connu. Il est dominé par l'insuffisance en protides des pâturages naturels, - surtout constitués de graminées, - qui entraine la nécessité d’un apport azoté complémentaire (9). Celui-ci est fait essentiellement sous forme de tourteau d'arachide, c'est-à-dire qu'il est peu diversifié. De plus, on sait que l'équilibre en acidesaminés des protides de l'arachide est loin d'être parfait, puisqu'on $y$ relève un déficit très marqué en méthionine, en isoleucine et en thréonine.

Parmi les produits d'origine animale, les farines de poisson sont mieux équilibrées, mais l'éleveur n'en trouve sur le marché que de façon très irrégulière. De plus, les farines de poisson qu'il peut se procurer sont, dans la presque totalité des cas, des farines d'importation dont le prix de revient est élevé.
Or, Madagascar dispose d'une faune de crustacés, - tant d'eau douce que d'eau de mer, abondante et très variée, et l'extension de l'exploitation de celle-ci est susceptible de fournir un appoint appréciable de protides aux animaux d'élevage.

Parmi ces crustacés, on trouve couramment sur le marché des crevettes de petite taille, séchées dès la capture, et appelées PATSA. Celles-ci sont utilisées actuellement exclusivement pour lalimentation humaine; elles sont consommées entières, avec la carapace, après cuisson. L'abondance de ces petites crevettes laisse entrevoir une possibilité d'utilisation de ce produit en alimentation animale.

D'autre part, la pêche de grosses crevettes de mer se pratique sur la Côte Ouest de Madagascar, dans la région de Majunga. Ces crevettes sont conditionnées en vue de l'exportation (élimination des céphalothorax, calibrage et congélation). Cette préparation industrielle 
laisse un tonnage très important de déchets (plusieurs centaines de tonnes de céphalothorax par an). La récupération de ces déchets pour l'alimentation animale semble, a priori, intéressante.

Le but de notre étude a été de déterminer dans quelle mesure ces produits locaux (petites crevettes entières et céphalothorax de grosses crevettes) pouvaient remplacer les farines de poisson d'importation, et pouvaient en particulier apporter aux animaux d'élevage (notamment aux porcs et aux volailles) les acides-aminés qui leur sont nécessaires.

\section{PETITES CREVETTES}

Ce sont des crevettes de petite taille, longues de 2 à $3 \mathrm{~cm}$ environ à l'état adulte, et séchées au soleil sur des nattes dès leur capture. Il en existe deux variétés commerciales : les crevettes « rouges » (Patsa mena) et les crevettes « blanches » (Patsa fotsy).

On les pêche généralement à l'aide de nasses traînantes souples ou de filets moustiquaires $(6,7,8)$.

a) Les crevettes " rouges » sont des crevettes d'eau douce. On les trouve dans la plupart des plans d'eau de Madagascar. Ce sont des Atyidae dont il existe plusieurs variétés. Celles que nous avons analysées sont des Caridina nilotica xiphias, Bouvier.

Ces crevettes sont blanches au moment de leur capture. C'est pendant le séchage au sole1l que leur carapace prend une teinte rouge (d'où leur nom vernaculaire). Celles qui sont vendues à Tananarive proviennent surtout de la région de Didy (marais au sud du Lac Alaotra).

Le long de la Côte-Est de Madagascar, leur pêche fait l'objet d'une technique particulière, basées sur les mœurs diurnes de ces crustacés : des «vovomora» (pièges constitués par des fougères retenues par des piquets) sont disposés dans les eaux de faible profondeur. Les crevettes (ainsi que des petits poissons) viennent s'y réfugier la nuit. Leur capture se fait très tôt, le matin. On utilise également sur la Côte-Est des petits barrages de branchages (vilasilaka), légèrement obliques par rapport à la berge, aux extrémités desquels sont disposées des nasses qu'on relève tôt le matin. b) Les crevettes «blanches» sont des crevettes de mer. Ce sont des Penaeidae. Celles que nous avons analysées sont des Sergestes arcticus, Kröyer. Elles sont également séchées au soleil et commercialisées sous cette forme.

Nos analyses ont porté sur des échantillons de crevettes d'eau douce, et de crevettes de mer (crevettes entières avec céphalothorax et carapace, c'est-à-dire telles qu'elles sont utilisées dans l'alimentation).

\section{CEPHALOTHORAX DE GROSSES CREVETTES}

Nous avons vu que ce produit constitue les déchets de la préparation industrielle de grosses crevettes de mer.

Seules sont «étêtées 》 les crevettes pêchées par chalutage dans les fonds de faible profondeur de la région de Majunga. Les espèces pêchées sont constituées par 80 p. 100 de Penaeus indicus, et 20 p. 100 de mélange de Penaeus monodon, Penaeus japonicus, Metapenaeus monoceros et Penaeus semisulcatus (*) $(1,3,7)$.

Par suite du manque d'installations appropriées (séchoirs, broyeurs...) ces déchets ne sont pas traités actuellement. Ils s'altèrent rapidement et doivent être rejetés à la mer. Des essais de séchage au soleil ont déjà été faits, mais sans résultats probants. Il est vrai que ceux-ci avaient été effectués pendant la saison humide (*).

Il serait souhaitable qu'un procédé économique de dessiccation puisse être utilisé dans un proche avenir pour permettre la récupération de ces tonnages importants de déchets.

Nos analyses ont été effectuées sur des échantillons de céphalothorax de crevettes, congelés pour le transport.

\section{ETUDE BIOCHIMIQUE}

Cette étude a porté :

$1^{\circ}$ Sur la composition biochimique globale (selon les méthodes officielles d'analyse des échantillons de produits de l'alimentation anjmale).

(*) Renseignements fournis par le Service de la Pêche Maritime-Tananarive. 
Les résultats figurent dans le tableau $\mathbf{I}$.

TABLEAU I

Composition chimique des echantillons utilisës jour le dosage des acides amınês (p.100 de produit sec)

\begin{tabular}{|c|c|c|c|}
\hline Composition & Caniona nilotica & Sergestes arctious & Cephalothorax de crevettes \\
\hline Matières minérales & 19,65 & 18,59 & 25,59 \\
\hline Matières grasses & 17,64 & 6,63 & 6,50 \\
\hline Yatières azotées & 60,48 & 73,64 & 58,74 \\
\hline Insoluble chlorhydrique & 5,66 & 3,95 & 0.51 \\
\hline Phosphore (en P) & 0,93 & 1,13 & 1,35 \\
\hline Calcium (en Ca) & 3,75 & 3,03 & 7,47 \\
\hline Clilorures (en NaCl) & 0,71 & 3,31 & 2,98 \\
\hline $\begin{array}{l}\text { Lau pour } 100 \text { g de } \\
\text { produit brut }\end{array}$ & 9,25 & 11,75 & 73,64 \\
\hline
\end{tabular}

$2^{\circ}$ Sur la composition en acides-aminés (par chromatographie sur colonne de résine, à l'exception du Tryptophane, dosé par la méthode de FISCHL modifiée). Les références des techniques employées ont été données dans un précédent article (4).
Les résultats figurent dans le tableau II.

Pour apprécier la valeur nutritive des protides étudiés, nous avons inscrit également dans ce tableau la composition moyenne d'une farine de poisson, d'après diverses données tirées de la littérature $(2,5)$.

\section{TABLEAU II}

Composition en acides aminés

(Résultats exprimés sur la base de $\mathrm{N}=16 \mathrm{p} .100$ )

\begin{tabular}{|c|c|c|c|c|}
\hline & Protides de référence & & Produits analysês & \\
\hline & Farine de poisson & Canidina nitotiad & Sergestes arcticus & $\begin{array}{l}\text { Céphalothorax } \\
\text { de crevettes }\end{array}$ \\
\hline Cystine & 1,1 & 1,16 & 1,24 & 0,70 \\
\hline Acide aspartique & 10,0 & 11,45 & 11,14 & 9,70 \\
\hline Thréonine & 4,3 & 4,58 & 4,52 & 4,24 \\
\hline Sërine & 4,9 & 4,38 & 4,13 & 4,23 \\
\hline Acide glutamique & 13,1 & 13,43 & 14,81 & 13,37 \\
\hline Proline & 5,3 & 4.60 & 4,33 & 5,74 \\
\hline Glycine & 6,9 & 5,18 & 7,80 & 6,38 \\
\hline Alanine & 6,6 & 6,53 & 6.76 & 6,54 \\
\hline Valine & 6,0 & 5.37 & 4,68 & 5,24 \\
\hline Mêthionine & 2,9 & 2,92 & 2,96 & 2,32 \\
\hline Iso-leucine & 5,2 & 4,88 & 4,25 & 4,14 \\
\hline Leucine & 7,2 & 6,92 & 7,28 & 6,38 \\
\hline Tyrosine & 2,9 & 4,06 & 3,53 & 3,62 \\
\hline Pbény1-alanine & 3,9 & 4,40 & 4,58 & 4,34 \\
\hline Lysine & 8,8 & 6,76 & 7,40 & 5,43 \\
\hline Histidine & 2,5 & 2,28 & 1,75 & 1,66 \\
\hline Tryptophane & 1,1 & 1,20 & 0,94 & 0,85 \\
\hline Arginine & 6,8 & 7,32 & 7,86 & 5,43 \\
\hline
\end{tabular}




\section{OBSERVATIONS \\ SUR LA COMPOSITION CHIMIQUE DES CREVETTES}

Sur les divers échantillons de crevettes que nous avons été amenés à analyser jusqu'ici, nous avons pu faire les constatations suivantes (les résultats ci-après sont exprimés, sauf indications contraires, pour $100 \mathrm{~g}$ de matière sèche totale) :

\section{Matières minérales}

Celles-ci sont relativement élevées pour les 3 produits (de 17,3 à 32,1 pour les petites crevettes et de 25,6 à 26,7 pour les céphalothorax de grosses crevettes). Mais elles contiennent une surcharge très variable de cendres insolubles dans l'acide chlorhydrique (silice), constituée vraisemblablement par du sable.

L'insoluble chlorhydrique trouvé pour les petites crevettes varie, selon les échantillons, de 0,9 à 18,4 p. 100 de la matière sèche. Pour les céphalothorax de grosses crevettes, ces taux sont beaucoup plus faibles (de 0,1 à 0,6 p. 100).

Déduction faite de cet insoluble chlorhydrique, les 2 variétés de petites crevettes contiennent de 12,5 à 16,4 p. 100 de matières minérales. Les céphalothorax de crevettes en contiennent beaucoup plus (de 25,1 à 26,1 p. 100).

\section{Phosphore}

Les 3 produits contiennent une quantité appréciable de phosphore. Nous avons noté les teneurs suivantes (exprimées en $P$ ) :

- Petites crevettes d'eau douce de 0,9 à 1,1 p. 100

- Petites crevettes de mer de 1,1 à 1,7 p. 100

- Céphalothorax de grosses crevettes

de 1,4 à 1,9 p. 100

\section{Calcium}

Les 3 produits constituent une source intéressante de calcium. Les petites crevettes de mer sont relativement les moins riches : de 2,9 à 3,1 p. 100 exprimé en $\mathrm{Ca}$. Les petites crevettes d'eau douce en contiennent de 3,8 à 5,2 p. 100. Les céphalothorax de grosses cre- vettes sont nettement plus riches : de 7,5 à 8,1 p. 100 .

\section{Chlorures}

La teneur en chlorures (exprimée en $\mathrm{ClNa}$ ) marque l'origine de ces produits :

- Petites crevettes

" rouges 》 (crevettes

d'eau douce) $\quad 0,7$ p. 100

- Petites crevettes

blanches (crevettes

de mer)

de 1,9 à 3,3 p. 100

- Céphalothorax

(de crevettes

de mer) de 1,8 à 3,0 p. 100

\section{Matières grasses}

Les teneurs trouvées sont variables pour les différents échantillons :

- Petites crevettes d'eau douce de 11,3 à 17,6 p. 100

-- Petites crevettes de mer

de 2,9 à 6,6 p. 100

- Céphalothorax de grosses crevettes de 5,6 à 9,3 p. 100

Les taux élevés de matières grasses risquent de nuire à la bonne conservation du produit.

\section{Protides}

Pour mieux comparer les richesses en matières azotées des 3 produits, nous avons fait figurer dans le tableau suivant, leurs teneurs en protides totaux exprimées d'une part pour 100 parties de matière sèche totale, et d'autre part pour 100 parties de matière sèche privée de son insoluble chlorhydrique.

Les 3 produits constituent une excellente source de matières azotées, comparable, quantitativement, aux farines de poisson.

Ce sont les petites crevettes de mer qui sont les plus riches en protides.

Bien que possédant une teneur nettement moindre en protides totaux, les céphalothorax de crevettes n'en constituent pas moins une source azotée particulièrement intéressante. 


\begin{tabular}{|l|r|c|}
\hline $\begin{array}{c}\text { Teneur en protides } \\
(\text { N } \times 6,25)\end{array}$ & Pour 100 de matière sèche totale & $\begin{array}{c}\text { Pour 100 de matière sèche privée } \\
\text { de son insoluble chlorliydrique }\end{array}$ \\
\hline Petites crevettes d'eau douce & 60,5 à 64,8 & 64,1 à 65,4 \\
\hline Petites crevettes de mer & 61,7 à 73,6 & 72,6 à 76,7 \\
\hline Céphalothorax de grosses crevettes & 51,9 à 58,7 & 52,2 à 59,0 \\
\hline
\end{tabular}

\section{INTERPRETATION DES RESULTATS}

Nous avons comparé la composition en acides-aminés des protides de ces trois produits avec celle d'une farine de poisson (moyenne de divers résultats fournis par la littérature) et en avons tiré les observations suivantes:

\section{Caridina nilotica}

La teneur en lysine des protides de ce produit est nettement inférieure à celle d'une farine de poisson ( -23 p. 100). Par contre, celle de la tyrosine est beaucoup plus élevée $(+40$ p. 100). Les autres acides-aminés, dont la cystine, la méthionine et l'isoleucine, ont des teneurs sensiblement équivalentes.

\section{Sergestes arcticus}

Les teneurs en cystine et en méthionine des protides de ce produit sont comparables à celles d'une farine de poisson. La lysine est légèrement inférieure ( -16 p. 100), ainsi que l'iso-leucine (- 18 p. 100). La teneur en histidine est nettement inférieure $(-30$ p. 100) ainsi que la valine ( -22 p. 100). Par contre, les teneurs en tyrosine $(+22$ p. 100), phénylalanine $(+17$ p. 100$)$ et en arginine $(+16$ p. 100) sont supérieures.

\section{Céphalothorax de grosses crevettes}

Les protides de ce produit sont nettement moins riches que ceux d'une farine de poisson en ce qui concerne les acides-aminés soufrés (cystine : - 36 p. 100 , méthionine : -20 p. 100), la lysine (-38 p. 100), l'isoleucine ( -20 p. 100) et l'histidine $(-34$ p. 100). Ils sont par contre plus riches en tyrosine $(+25$ p. 100).
Bien qu'il soit moins équilibré qu'une farine de poisson, ce produit est cependant susceptible de fournir dans l'alimentation animale un apport important des principaux acides-aminés.

\section{CONCLUSION}

Les résultats de l'étude que nous avons faite sur les Caridina nilotica, les Sergestes arcticus et les céphalothorax de grosses crevettes montrent que ces 3 produits peuvent être substitués, pour l'alimentation animale, aux farines de poisson. Ils sont susceptibles de fournir aux animaux d'élevage un apport appréciable de protéines dont la composition en acides-aminés est, dans l'ensemble, satisfaisante. Ils constituent également une bonne source de phosphore et surtout de calcium dont les aliments traditionnels ne fournissent souvent qu'un apport insuffisant.

Il serait intéressant que la pêche de petites crevettes, tant d'eau douce que de mer, puisse être augmentée pour permettre, au moins dans les régions productrices, leur distribution aux porcs et aux volailles.

En ce qui concerne les céphalothorax de grosses crevettes qui représentent un tonnage considérable de déchets de l'industrie de la pêche sur la Côte-Ouest, il serait souhaitable qu'ils soient récupérés et conditionnés rationnellement en vue de l'alimentation animale, par exemple sous forme de farine, après séchage.

L'utilisation de ces produits serait enfin susceptible de limiter les nécessités d'importation de farines animales, ce qui constituerait par voie de conséquence, un avantage appréciable pour l'économie malgache.

Laboratoire Central de l'Elevage, Tananarive. 


\section{REMERCIEMENTS}

Nous tenons à adresser nos vifs remercie-

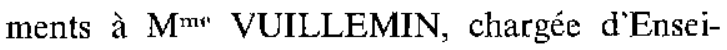
gnement à l'Université de Tananarive, pour l'identification des échantillons des petites crevettes de mer et d'eau douce que nous avons étudiées, ainsi qu'à M. VINCKE, IngénieurTechnicien des Pêches, du Centre Technique Forestier Tropical de Tananarive, pour les renseignements qu'il nous a fournis sur la pêche des crevettes d'eau douce.

Nous remercions vivement également Monsieur le Vétérinaire-Inspecteur BLANC, Chef du Service de la Pêche Maritime à Madagascar qui nous a approvisionnés en échantillons de céphalothorax de crevettes et qui nous a fourni également d'utiles renseignements sur ce produit.

\section{SUMMARY}

Note on the ammo-acid composition of shrimps usable for the feeding of breeding animals in Madagascar

Madagascar is well stocked with some crustacea that can be used in animal feeding, instead of imported fish meals :

- Little fresh-water shrimps: Caridina nilotica xiphias Atyidae.

- Little sea-water shrimps: Sergestes arcticus Penaeidae.

So it is with the cephalothorax of big sea-water shrimps (Penaess indicus) that are industrial fishing waste in the Majunga area.

The total chemical composition and the amino-acid composition of these 3 products are indicated in this article.

\section{RESUMEN}

Nota sobre Ia composición en aminoácidos de camarones que se pueden utilizar en la alimentación de los animales criados en Madagascar

Se podrían utilizar, en la alimentación animal, ciertos crustáceos encontrados numerosos en Madagascar, en lugar de harinas de pescado importadas :

- Camaroncillos de agua dulce: Caridina nilotica xiphias Atyidae.

- Camaroncillos de mar: Sergestes arcticus, Penaeidae.

Es igual para los cefalotorax de gruesas camarones de mar (Penaeus indicus...) que constituyen residuos de la pesca industrial en la región de Majunga.

Se indican, en esta nota, la composición química global y la composición en aminoácidos de dichos tres productos.

\section{BIBLIOGRAPHIE}

1. ANGOT, «Les applications de la recherche océanographique à Nossy-Be», Bull. Madagascar, 1968, 18 (264) : 458-63.

2. BLOCK (R. J.) et WEISS (K.W.), « Amino-acid handbook », Thomas, 1956.

3. CROSNIER (A.), CHARBONNIER (D.), et LAGOIN (Y)., «Quelques données sur la pêche à la crevette à Madagascar », Bull. Madagascar, 1960, (175) : 1099.

4. GAULIER (R.), «Composition en acides-aminés des principales légumineuses fourragères de Madagascar ", Rev. Elev. Méd. vét. Pays trop., 1968, 21 (1): $103-112$.
5. JACQUOT (R.), LEBARS (H.) et SIMONNET (H.), «Données générales sur la nutrition et l'alimentation », Paris, J. B. Baillière, 1958.

6. KIENER (A.), «La pêche au piège ou Voyomora dans les Pangalanes-Est », Bull. Madagascar, 1960, (167) : 309-14.

7. KIENER (A.), «Poissons, pêche et pisciculture à Madagascar », Nogent sur Marne, Centre Technique Forestier Tropical, 1963. Publication No 24.

8. MOULHERAT (J. L.) et VINCKE, « Etude en vue du développement de la pêche aux Pangalanes-Est (Zone Tamatave - Andevoranto) », Nogent sur Marne, Centre Technique Forestier Tropical, 1963. 9. SERRES (H.), "Eléments d'alimentation du bétail à Madagascar», Tananarive, I.E.M.V.T., 1966. 